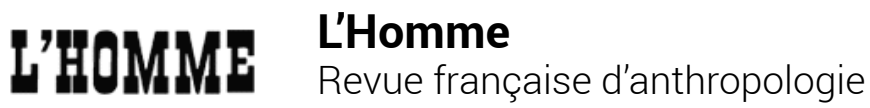

187-188 | 2008

Miroirs transatlantiques

\section{Mondher Kilani, Guerre et sacrifice. La violence extrême}

Wiel Eggen

\section{(2) OpenEdition}

1 Journals

\section{Édition électronique}

URL : https://journals.openedition.org//homme/20862

DOI : $10.4000 /$ /homme.20862

ISSN : 1953-8103

Éditeur

Éditions de l'EHESS

\section{Édition imprimée}

Date de publication : 3 octobre 2008

Pagination : 510-512

ISBN : 978-2-7132-2186-6

ISSN : 0439-4216

\section{Référence électronique}

Wiel Eggen, « Mondher Kilani, Guerre et sacrifice. La violence extrême », L'Homme [En ligne], 187-188|

2008, mis en ligne le 16 décembre 2008, consulté le 24 avril 2022. URL : http://

journals.openedition.org//homme/20862 ; DOI : https://doi.org/10.4000//homme.20862

Ce document a été généré automatiquement le 24 avril 2022.

(c) École des hautes études en sciences sociales 


\title{
Mondher Kilani, Guerre et sacrifice. La violence extrême
}

\author{
Wiel Eggen
}

\section{RÉFÉRENCE}

Mondher KILANI, Guerre et sacrifice. La violence extrême. Paris, Presses universitaires de France, 2006, 140 p., bibl., index (« Ethnologies-Controverse »).

1 Peut-on ranger sous la même étiquette les «dommages collatéraux " causés par des missiles sophistiqués et les attaques-suicides des terroristes, et peut-on faire appel à la notion de sacrifice pour penser ensemble ces formes de violence extrême? Le livre de Mondher Kilani tente cette gageure, et il est d'autant plus déconcertant qu'il mêle analyse anthropologique et combat politique. Ceux qui aiment les distinctions nettes et croient possible de garder, en ces matières, une neutralité scientifique, lui reprocheront de brouiller les catégories et d'amalgamer jugements de fait et jugements de valeur. Lorsque, sous les yeux d'une famille musulmane, qui n'a pas encore tué le mouton de l'Aïd, un soldat serbe égorge un adolescent kosovar, en disant que ce dernier constituera une meilleure offrande, puisque Abraham avait reçu l'ordre d'immoler Ismaël (p. 94), il témoigne d'une certaine proximité de la guerre et du sacrifice, mais aussi de leur différence radicale. Car, pour lui comme pour sa victime, il est clair qu'il s'agit d'un crime doublé d'un sacrilège. Au moins cet exemple, qui rappelle les origines violentes du sacrifice, permet-il de conjecturer une proximité étrange, voire un rapport intime, entre pratiques religieuses et pratiques de guerre. Mais, y a-t-il quoi que ce soit de commun entre cet acte para-sacrificiel et les sévices effectués par des soldats américains sur les prisonniers irakiens d'Abou Graib ou encore certains traitements humiliants infligés à Saddam Hussein? Par ailleurs, est-il objectif de présenter les impérialistes américains comme des ennemis des peuples libres, particulièrement musulmans, sans rappeler qu'ils ont organisé la défense du Kosovo contre les Serbes? Ou encore, est-il bien légitime de qualifier d'acte impérialiste l'occupation du Japon en 1945, et de fermer les yeux sur le massacre des Kurdes et l'invasion du Koweit par 
Saddam, au motif que celui-ci servirait de bouc émissaire aux impérialistes? Enfin, n'est-il pas risqué de réduire le sida à une maladie manipulée à des fins d'hégémonie par les puissances occidentales?

2 Le livre de Kilani pose malgré tout un vrai problème de fond. Il s'agit de savoir si la guerre et le sacrifice, ainsi d'ailleurs que d'autres types de violence institutionnelle, telle la vendetta, obéissent bien à des logiques fondamentalement différentes, comme le pensent la plupart des anthropologues, et peuvent se perpétuer en conservant leur hétérogénéité. Ou si, au contraire, toutes ces différences ne seraient pas superficielles, et ne tendraient pas à s'effacer dans le monde moderne "globalisé » où de multiples formes de violence, qu'elles soient d'ailleurs codifiées ou sauvages, mais toujours massives et acceptées dans une sorte d'indifférence générale, semblent avoir pour trait commun une «tonalité sacrificielle qui ne se déclare pas » (pp. 13 et 108).

Que faut-il entendre par là ? Bien que notre auteur ne donne pas de définition précise du sacrifice, l'usage extensif qu'il fait du terme repose, à l'évidence, sur l'observation suivante. Un trait caractéristique du sacrifice proprement dit, qu'il soit humain ou animal, est d'autoriser les hommes à faire un grand nombre de victimes dans une sorte d'apathie générale, au nom d'un objectif moral supérieur (p. 101). Alors que, dans la vie ordinaire, la mise à mort, ou seulement les mauvais traitements, infligés aux hommes ou aux animaux soulèvent l'indignation de tous, dans le contexte sacrificiel, ces violences sont acceptées. Il faut bien, croit-on, pour honorer les esprits ou les dieux, ou célébrer les fêtes destinées à perpétuer la société, accomplir de telles violences. Or, remarque Kilani, il y a des situations apparemment profanes, où l'on observe la même indifférence au sort des victimes. Un exemple simple et probant serait celui des accidents de la route. Jusqu'à ces toutes dernières années, tout le monde ou presque acceptait, notamment en France, que «la route»-comme on disait pour dégager la responsabilité des hommes - «fit chaque année dix mille victimes », soit beaucoup plus que les dernières guerres dans lesquelles le pays s'était trouvé engagé. Ces morts étaient comme un sacrifice ou un tribut payé au dieu automobile, ou encore un « dommage collatéral » du progrès technique. On les tenait, et on les tient encore, pour inévitables, alors que, en réduisant drastiquement la vitesse des véhicules, on ferait tendre vers zéro le nombre de victimes. Reste à savoir si ce raisonnement peut s'appliquer à tous les exemples proposés par Kilani (de l'abattage massif des animaux en cas d'épizootie, à l'attribution au virus du sida d'une maladie d'abord causée par la misère et la pauvreté), et aboutir à la conclusion générale, reprise de Bernard Lempert (2000), selon laquelle, loin d'endiguer la violence et la bellicosité humaines comme le soutient René Girard, le schème sacrificiel permettrait au contraire de les légitimer, de les attiser, et de leur trouver sans cesse de nouveaux aliments.

4 Estimant, comme Clausewitz, que la guerre n'est que de la politique poursuivie avec d'autres moyens, Kilani contourne le modèle structuraliste du lien social - qui, en plaçant l'échange des maux, des violences et des rapines sur le même plan que celui des biens, des femmes et des dons, attribue une trop grande symétrie aux acteurs sociaux -, pour rejoindre la vue de Carl Schmitt suivant laquelle, en politique, il s'agit avant tout de distinguer les amis des ennemis. Mais il développe le schème bipolaire du philosophe allemand en fonction des trois cercles de violence repérés par Raymond Verdier, celui de l'identité, où la violence est interdite et ne peut être expiée que par des sacrifices, celui de l'adversité, où la violence est permise, voire prescrite, mais soumise aux règles du système vindicatoire, et celui enfin de l'hostilité, où tous les 
coups sont permis sans qu'il y ait, à proprement parler, de lois de la guerre. Comme Paul Dumouchel, il rattache les luttes inter-étatiques des temps modernes aux conflits traditionnels du deuxième cercle, mettant aux prises des pairs respectueux de leur identité mutuelle, pour leur opposer une nouvelle forme de violence, post-étatique, déjà entrevue par Schmitt, et caractérisée par le fait que, tout en appartenant à un même ensemble social, les opposants se voient les uns les autres comme engagés dans une lutte totale entre le Bien et le Mal. Dans cette perspective, on irait vers une situation de guerre civile généralisée, où il n'aurait plus dedans ni dehors, et qui, d'après Schmitt lui-même, aurait déjà commencé, autour de 1800, avec les guerres révolutionnaires, pour se développer avec les techniques militaires qui permettent de laisser les victimes hors de vue et de faire croire à de possibles combats extrêmes mais sans morts. La mission du politique serait ainsi devenue totalisante et guerrière, acceptant la pire violence pour le bien commun. Kilani montre que, dans ce contexte, la guerre est, de part et d'autre, presque toujours perçue en termes sacrificiels, - les puissants sacrifiant à distance, et les autres se sacrifiant en martyrs - mais sans que ces nouvelles formes de sacrifice puissent résoudre la moindre crise ni détruire l'ordre sacrificiel comme le sacrifice du Christ était supposé l'avoir fait. Bien pis, cette logique sacrificielle entretient la violence extrême en faisant disparaitre tout sens d'autrui. Au choc des civilisations (Huntington), Kilani oppose donc l'idée d'une guerre totale étendue à l'ensemble des hommes, à laquelle chacun prendrait part, mais à partir de positions différentes, au sein d'un processus générateur d'une nouvelle forme d'humanité (anthropopoèsis, p. 122). Toutes ces positions ayant en commun une même tonalité sacrificielle "qui ne se déclare pas ", le livre se clôt par un appel à rejeter la valorisation du religieux d'un Girard ou même d'un Hocart, pour choisir un projet politique résolument laïque, de type contrat social, comme le propose Gauchet (p. 129).

Kilani se situe dans la tradition des maîtres du soupçon, croyant comme eux que la logique sociale échappe aux acteurs ou permet aux puissants d'utiliser à leur profit des valeurs supposées transcendantes. Mais il en tire des conclusions nouvelles, en montrant dans quelle forme originale de guerre civile généralisée est entraîné le monde contemporain, ce monde supposé «désenchanté» ou "désacralisé », et pourtant pétri de schèmes sacrificiels. Il fait sienne, à cet égard, l'analyse girardienne du mécanisme victimaire et l'idée que celui-ci opère nécessairement dans la méconnaissance, mais sans croire un seul instant qu'une quelconque conversion religieuse (chrétienne ou autre) puisse jamais détourner le cours d'une logique sacrificielle qui entraîne progressivement l'humanité dans une vaste société mondiale décloisonnée et en proie à une guerre intestine générale, où les affrontements prennent la forme d'une lutte sans merci entre le Bien et le Mal, justifiant l'élimination d'individus "sacrifiables", tant dans les affrontements armés que dans les luttes économiques.

Mais, si stimulante que soit cette analyse, elle comporte des points d'ombre. On peut craindre que l'auteur, qui se refuse à donner une définition précise du sacrifice (p. 89), ne fasse de cette notion un usage si lâche qu'elle lui retire tout contenu spécifique. Par ailleurs, la notion de « contrat social », qu'il lui oppose (p. 128), peut-elle constituer une alternative crédible, alors qu'elle a, d'entrée de jeu, partie liée avec le projet d'une maîtrise totale de la société par la Raison et les luttes révolutionnaires - et « sacrificielles », elles aussi - pour le triomphe du Bien ? Sur quoi pourrait-on fonder un contrat social, sinon sur une base qui ne dépend pas des contractants et les invite à s'entendre? Autrement dit sur les exigences rituelles et religieuses analysées par un 
Hocart ou un Girard que Kilani croit pouvoir récuser ? «La moralité, soutenait déjà Chesterton, n'est pas née du jour où un homme a dit à un autre : "Je ne te frapperai pas si tu ne me frappes pas" [...] Mais il y a eu deux hommes pour dire: "Nous ne devons pas nous frapper dans ce lieu sacré". Ils ont acquis leur morale en défendant leur religion ». On peut penser, avec Lucien Scubla, qu'il n'y a pas d'autre forme possible de contrat social. En dépit de ses faiblesses, le livre nous engage à un débat fondamental, digne de la collection «Ethnologies-Controverses ». Il est bien écrit, doté d'un index très élaboré. 